\title{
An Extended-Range Incremental CT $\Sigma \Delta$ ADC with Optimized Digital Filter
}

\author{
Julian Garcia ${ }^{1}$ and Ana Rusu \\ School of Information and Communication Technology (ICT) \\ Royal Institute of Technology (KTH) \\ SE-164 40 Kista, Sweden \\ ${ }^{1}$ E-mail: julianmg@kth.se
}

\begin{abstract}
Extended range approach has been employed in discretetime incremental sigma-delta analog-to-digital converters to reduce the number of cycles per conversion and therefore the power dissipation. In this work, extended range is combined with continuous-time filter implementation so as to reduce the integrators gain-bandwidth product requirement. The proposed architecture and mathematical analysis are presented using a $3^{\text {rd }}$ order single-loop single-bit sigma-delta modulator as proof-ofconcept. In order to overcome the analog-digital transfer functions mismatches, an appropriate digital filter is designed using optimization tools. Behavioral simulations show that the proposed architecture with an optimized filter achieves 13.8 bits resolution with a $4 \mathrm{kSamples} / \mathrm{sec}$ sampling rate to comply with a high-resolution biomedical application.
\end{abstract}

\section{Keywords}

A/D conversion, extended-range sigma-delta ADC, continuoustime.

\section{Introduction}

Today analog-to-digital converters (ADCs) are increasingly being integrated into time-multiplexed low-power highresolution biosensor applications, such as wearable and implantable biomedical systems [1-3]. While the required bandwidth is generally relaxed, the required resolution is, on the other hand, more stringent and varies from 12 to 16 bits, depending on each specific application. These requirements have been successfully covered by incremental sigma-delta $(\mathrm{I} \Sigma \Delta)$ [1,4-6] and extended-range (ER) I $\Sigma \Delta$ ADCs [7]. From a power consumption perspective, single-loop (SL) high-order ER-I $\Sigma \Delta$ ADCs are especially attractive as they reduce the required number of cycles per conversion when compared to incremental counterparts. So far, discrete time (DT) implementations have been the main focus in ER-I $\Sigma \Delta$ ADCs while little attention has been paid to continuous-time (CT) counterparts. Continuous time implementation has been used, on the other hand, in traditional $\Sigma \Delta$ ADCs for low bandwidth applications [8], resulting in a power dissipation reduction. Such power reduction originates mainly from the relaxed slew rate and bandwidth requirements of the active blocks when compared to switch-capacitor counterparts. Moreover, even though a CT implementation has the potential of reducing the integrators gain-bandwidth product $(\mathrm{GBW})$ requirement, its

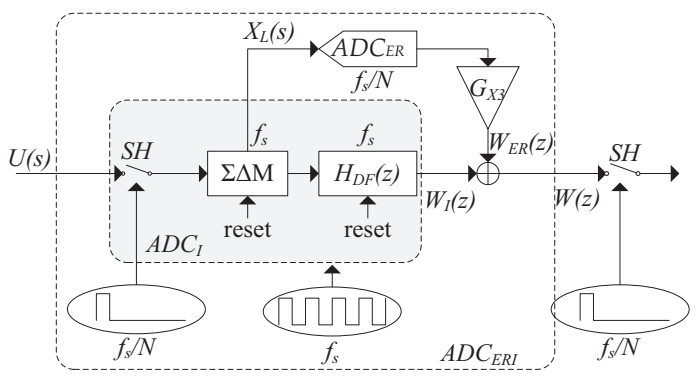

Figure 1: Generic SL ER-I $\Sigma \Delta$ ADC block diagram.

non-idealities also introduce new challenges. Particularly important for ER architectures is the degradation of the matching required between analog and digital transfer functions, which directly affects the ADC performance.

This work explores the challenges and opportunities of CT ER-I $\Sigma \Delta$ ADCs by means of a test-case targeting a highresolution ( $\geq 13$ bits) clinical electro-encephalogram (EEG) recording system. Section 2 begins by analyzing the proposed test-case operation along with its theoretical performance. Section 3 investigates the sensitivity to critical non-idealities, such as excess loop delay (ELD), clock jitter, integrator's coefficients deviation and finite's amplifier GBW product, and discusses their effect on the analog and digital transfer function mismatch. Taken this into account, Section 4 proposes the design of an appropriate digital filter by using optimization tools so as to minimize such mismatches. A final test case, when both critical non-idealities and the novel filter are included, is presented in Section 5. Finally, Section 6 concludes the paper.

\section{Proposed ER-I $\Sigma \Delta$ ADC}

I $\Sigma \Delta$ ADCs differ from traditional $\Sigma \Delta$ ADCs in that their memory elements are reset every time a new conversion takes place. This provides a one-to-one mapping between input and output and makes the I $\Sigma \Delta \mathrm{ADC}$ suitable for conversion of timemultiplexed signals [1]. As shown on the shaded area of Figure 1, a new conversion begins by sampling the input signal $U(s)$ and resetting the states of both the $\Sigma \Delta$ modulator and the digital filter $H_{D F}(z)$. The input signal is then hold for a period of $N / f_{s}$ while the ADC performs the conversion at $f_{s}$ frequency, where $N$ is the number of cycles per conversion and $f_{s}$ is the modulator's sampling frequency. After $N$ cycles have passed, a valid result is obtained from the output of the digi- 


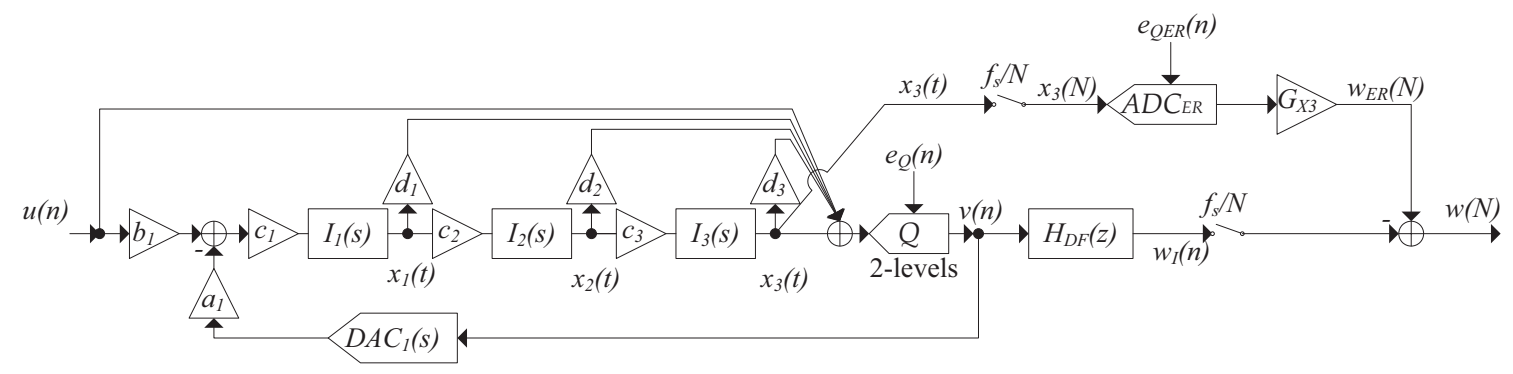

Figure 2: Block diagram of the proposed SL CT ER-I $\Sigma \Delta$ ADC.

tal filter $H_{D F}(z)$ and a new conversion can take place. Another particular characteristic of I $\Sigma \Delta$ ADCs is that the ADC quantization error can be made available at the last integrator's output when an appropriate filter is used. This feature is used in the extended range approach to improve the ADC resolution and, as a consequence, to reduce the required number of cycles per conversion, $N$. The quantization error refinement is obtained by adding a Nyquist-rate ADC, such as a successiveapproximation register (SAR) ADC [7], to capture the output of the last integrator, $X_{L}(s)$, so as to combine it with the $\mathrm{I} \Sigma \Delta$ ADC output, as shown on the top section of Figure 1. It is worth to notice that, even though an extra ADC has to be used, it operates at the decimated frequency, so its power dissipation may be very low [9]. Reducing the necessary number of cycles per conversion reduces, in turn, the sampling frequency $f_{s}$, which relaxes the integrator's GBW product requirements and may lead to lower power dissipation than CT I $\Sigma \Delta$ counterparts.

A block diagram of the proposed CT ER-I $\Sigma \Delta$ ADC is shown in Figure 2. A $3^{\text {rd }}$ order loop filter and cascade-ofintegrators in feed-forward (CIFF) configuration are used to respectively reduce the number of necessary cycles and the signal swing in the integrators path. Moreover, a single-bit quantizer has been chosen as it minimizes the digital filter complexity. Finally, a switched-capacitor-resistor (SCR) [10] digital-to-analog converter (DAC) is employed to reduce the sensitivity to jitter and ELD.

The design of a digital filter that would allow quantization error refinement in CT I $\Sigma \Delta$ ADCs was derived mathematically in [11]. This filter can then be used in order to obtain the theoretical performance of this architecture and is given by (1) where $a_{1}$ and $b_{1}$ are loop filter coefficients and $\tau$ is the mean lifetime value of the SCR-DAC. Similarly, the digital gain at the output of the extended range $\mathrm{ADC}, \mathrm{ADC}_{\mathrm{ER}}$, is given by:

$$
G_{X_{3}}=\frac{e_{Q-I}(N)}{x_{3}(N)}=\frac{6}{b_{1} c_{1} c_{2} c_{3} N^{3}}
$$

where $e_{Q-I}(N)$ is the quantization error of the $\mathrm{I} \Sigma \Delta \mathrm{ADC}$,

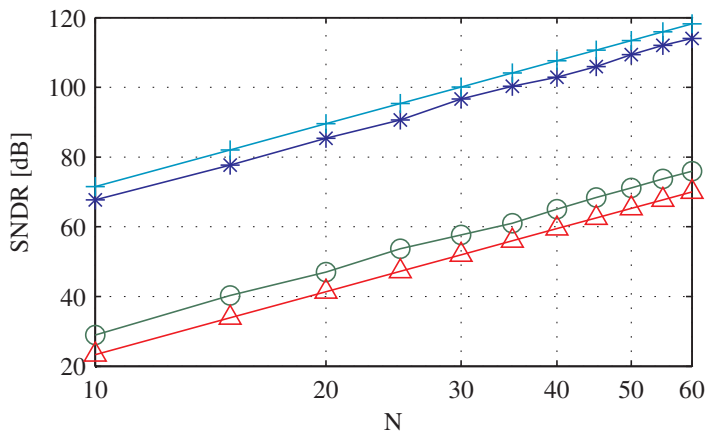

Figure 3: Simulated SNDR of ER-I $\Sigma \Delta(*)$ and I $\Sigma \Delta(\bigcirc)$ ADC against theoretical SNDR derived from (5) of ER-I $\Sigma \Delta(+)$ and $\mathrm{I} \Sigma \Delta(\triangle)$ ADC vs. number of cycles $(N)$. Input signal power: $P_{\text {sig }}=-6 \mathrm{dBFS}$.

$\mathrm{ADC}_{\mathrm{I}}$ at instant $N, x_{3}(N)$ is the sampled value of the $3^{\text {rd }}$ integrator's output at instant $N$, and $c_{1}, c_{2}$ and $c_{3}$ are also loop filter coefficients. As the extended range approach refines the quantization noise of the incremental $\mathrm{ADC}, \mathrm{ADC}_{\mathrm{I}}$, its leastsignificant-bit (LSB) quantization error can be expressed as:

$$
V_{L S B, E R}=\frac{V_{L S B, I}}{2^{B_{E R}}}
$$

where $B_{E R}$ is the number of bits in the extended range ADC, $\mathrm{ADC}_{\mathrm{ER}}$, and $V_{L S B, I}$ is the LSB quantization error of the incremental ADC. $V_{L S B, I}$ is obtained assuming the maximum range for the output of the $3^{\text {rd }}$ integrator equal to the maximum input full-scale value [4], $\pm U_{\mathrm{FS}}$, given by:

$$
V_{L S B, I}=2 U_{\mathrm{FS}} G_{X_{3}}=\frac{12 U_{\mathrm{FS}}}{b_{1} c_{1} c_{2} c_{3} N^{3}}
$$

The effective number of bits (ENOB) for the ER-I $\Sigma \Delta$ ADC, $\mathrm{ADC}_{\mathrm{ERI}}$, when a differential input signal with amplitude

$$
H_{D F}(z)=\left(\frac{\frac{1}{8}\left(8 \tau^{2}\left(1-\frac{1}{\mathrm{e}^{\frac{1}{2 \tau}}}\right)-4 \tau+1\right)}{(z-1)}+\frac{\frac{1}{2}\left(2 \tau\left(1-\frac{1}{\mathrm{e}^{\frac{1}{2 \tau}}}\right)-2+\frac{1}{\mathrm{e}^{\frac{1}{2 \tau}}}\right)}{(z-1)^{2}}-\frac{\left(1-\frac{1}{\mathrm{e}^{\frac{1}{2 \tau}}}\right)}{(z-1)^{3}}\right) \frac{6 a_{1}}{N^{3} b_{1}}
$$


$\pm U_{\text {max }}$ is considered, can then be expressed as:

$$
\begin{aligned}
\mathrm{ENOB}_{\mathrm{ERI}} & =\log _{2}\left(\frac{2 U_{\max }}{V_{L S B, E R}}\right) \\
& =\log _{2}\left(\frac{2 U_{\max }}{V_{L S B, I}} \cdot 2^{B_{E R}}\right) \\
& =\mathrm{ENOB}_{\mathrm{I}}+\mathrm{ENOB}_{\mathrm{ER}}
\end{aligned}
$$

where $\mathrm{ENOB}_{\mathrm{I}}$ is the $\mathrm{ENOB}$ of the $\mathrm{ADC}_{\mathrm{I}}$ and $\mathrm{ENOB}_{\mathrm{ER}}$ is the ENOB of the $\mathrm{ADC}_{\mathrm{ER}}$. According to (5), the ENOB of the $\mathrm{ADC}_{\mathrm{I}}$ is improved by $B_{E R}$ bits when extended range is applied. In theory, this can be a significant improvement, depending on the resolution of the $\mathrm{ADC}_{\mathrm{ER}}$. In practice however, two factors will contribute to the degradation of such theoretical performance. The first factor originates from the assumption that the output of the third integrator is equal to the maximum input full-scale value while in reality it might be less than such value. As this assumption is considered when calculating the $V_{L S B, I}$ value in (4) and the input range of the $\mathrm{ADC}_{\mathrm{ER}}$, it will affect both the performance of the I $\Sigma \Delta$ and ER-I $\Sigma \Delta$ ADC. With respect to $\mathrm{I} \Sigma \Delta \mathrm{ADC}$, its performance will be underestimated as the quantization noise is less than the one accounted by (4). On the other hand, the performance of the ER-I $\Sigma \Delta$ ADC will be overestimated as the $\mathrm{ADC}_{\mathrm{ER}}$ is not benefiting from its full dynamic range. These effects can be appreciated in Figure 3, where the signal-to-noise-plus-distortion-ratio (SNDR) performance of both the I $\Sigma \Delta$ and the ER-I $\Sigma \Delta$ ADCs were simulated while sweeping the number cycles $N$ and compared against their theoretical SNDR derived from (5). The second factor that will affect the theoretical performance is the degradation due to non-idealities. This is critical for the optimum performance of the ADC and will be analyzed in detail in the following sections.

\section{Nonideal behavior}

As it can be seen from (1) and (2), the quantization error refinement depends on the matching between a digital and an analog transfer function. This type of requirement is also found in cascaded $\Sigma \Delta$ ADCs [12] and special attention has to be paid to the mismatch caused by CT non-idealities as it would cause a leakage of the modulator's quantization noise to the output. In order to quantify the ADC performance degradation, MATLAB transient simulations have been performed while computing the SNDR of both the output of the ER-I $\Sigma \Delta$ and the I $\Sigma \Delta$ ADC, $\mathrm{ADC}_{\mathrm{ERI}}$ and $\mathrm{ADC}_{\mathrm{I}}$ respectively, under different non-ideal conditions. The ADC was run for 40 cycles while using an 8-bit $\mathrm{ADC}_{\mathrm{ER}}$ and a digital filter as described by (1). A mean lifetime value $\tau=1 / 25 T_{s}$ was used in the feedback SCR-DAC when analyzing the sensitivity to process variations and to finite integrators GBW product, while simulations with $\tau=1 / 10 T_{s}$ were added to the ELD and jitter analysis.

When considering the sensitivity to process variations, it has been assumed that the RC product would suffer the same spread [13]. As shown in Figure 4, the extended range implementation is, as expected, highly sensitive to coefficients variations and a tuning circuitry should be added in order keep the degradation between acceptable limits. It is also worth to notice the different sensitivity of incremental and extended range implementations.

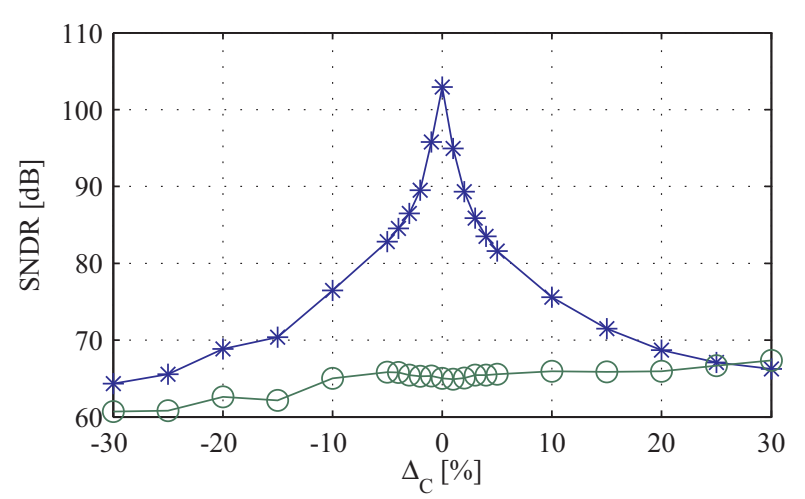

Figure 4: $\mathrm{ADC}_{\mathrm{ERI}}(*)$ and $\mathrm{ADC}_{\mathrm{I}}(\bigcirc)$ SNDR performance vs. integrators coefficients deviation. $P_{\text {sig }}=-6 \mathrm{dBFS}$.

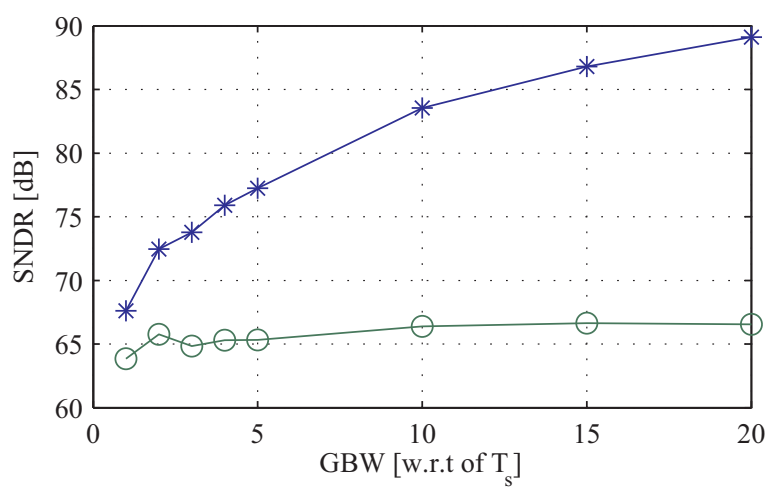

Figure 5: $\mathrm{ADC}_{\mathrm{ERI}}(*)$ and $\mathrm{ADC}_{\mathrm{I}}(\bigcirc)$ SNDR performance vs. integrators GBW product. $P_{\text {sig }}=-6 \mathrm{dBFS}$.

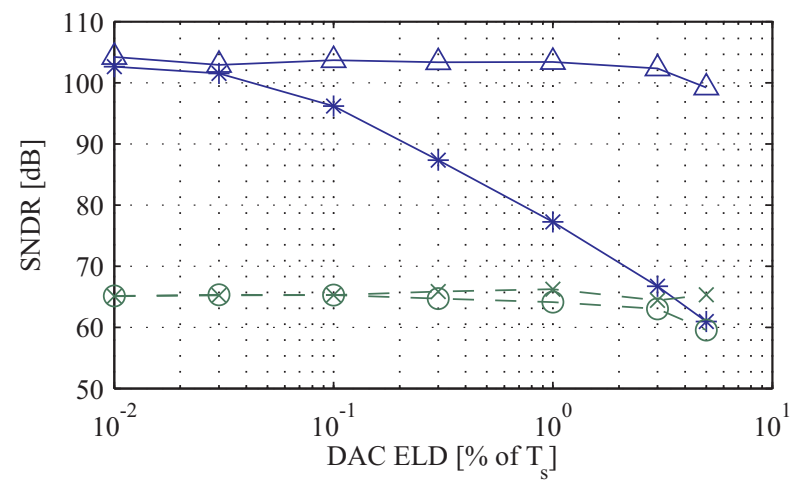

Figure 6: $\mathrm{ADC}_{\mathrm{ERI}}$ ('-') and $\mathrm{ADC}_{\mathrm{I}}$ ('- - -') SNDR performance vs. DAC ELD for different values of $\tau$. [ $* \& \bigcirc: \tau=1 / 10 T_{s}$, $\left.\triangle \& \times: \tau=1 / 25 T_{s}\right] . P_{\text {sig }}=-6 \mathrm{dBFS}$.

While on the former one the variation affects mainly the gain of $\mathrm{ADC}_{\mathrm{I}}$, thus having little impact on the SNDR, on the latter one it directly affects the $\mathrm{ADC}_{\mathrm{I}}$ quantization noise cancellation.

The effect of integrator's finite GBW product was studied by using single-pole models [14] and it is presented in Figure 5. Due to the analog-digital mismatches, it is not possible to benefit from the extended range approach when using a low GBW 


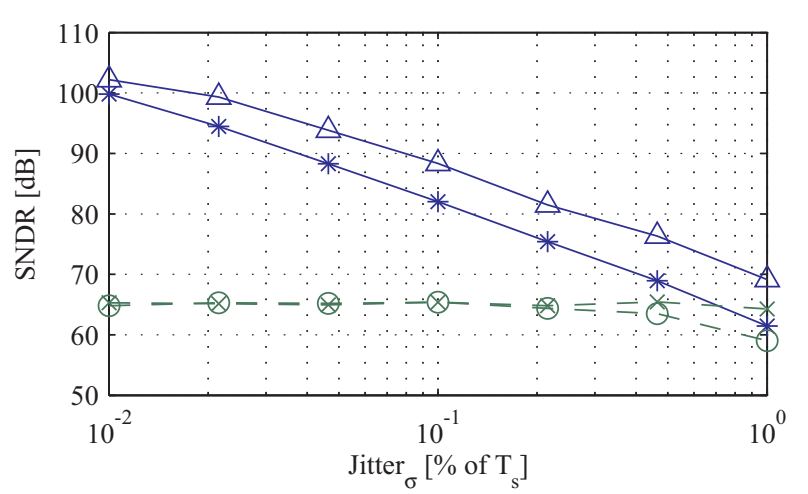

Figure 7: $\mathrm{ADC}_{\mathrm{ERI}}$ ('-') and $\mathrm{ADC}_{\mathrm{I}}$ ('---') SNDR performance vs. jitter standard deviation for different values of $\tau$. [ $* \& \bigcirc$ : $\left.\tau=1 / 10 T_{s}, \triangle \& \times: \tau=1 / 25 T_{s}\right] . P_{\text {sig }}=-6 \mathrm{dBFS}$.

product as its performance would approach to the incremental one. This reveals a severe drawback as it would directly impact the power consumption of the ER-I $\Sigma \Delta$ ADC.

Similarly to half-return-to zero (HRZ) coding scheme, the SCR scheme is active during the $2^{\text {nd }}$ half of the clock cycle. Therefore, as long as the quantizer delay is less than $0.5 T_{s}$, the ELD will be generated by the DAC delay only. As it can be seen from Figure 6, although the extended range architecture is more sensitive to ELD than the incremental counterpart, by choosing an appropriate mean lifetime value it is possible to counteract the error injected by the DAC delay. As in the previous case, the degradation due to clock jitter can also be counteracted by using an appropriate mean lifetime value, as can be seen in Figure 7.

The results of this section show an increase in the sensitivity to critical non-idealities of the extended range approach when compared to the incremental counterpart. This was expected as the extended range implementation performance is highly dependent on the matching between analog and digital transfer functions and therefore highlights the need of careful filter design to counteract such mismatches.

\section{Digital filter optimization}

Although several optimal filters have been derived for DT I $\Sigma \Delta$ ADCs $[5,15,16]$, to the authors' knowledge, the digital filter optimization for CT ER-I $\Sigma \Delta$ ADCs has not been treated yet. In this section, optimization tools are employed as an alternative approach to design such filter so as to counteract analogdigital mismatches.

As the transfer function mismatches stem from different non-idealities affecting the modulator, it would be possible, in principle, to mathematically derive a digital filter to account for such divergences. The main drawback of this approach is that it becomes too complicated when going from system level to more refined abstraction levels such as block level or circuit level implementation. The following approach, on the other hand, could be directly applied by just running the optimization algorithm in each of the design steps.

The digital filter matching the ideal analog transfer function given in (1) is a sum of cascade of integrators which process $N$

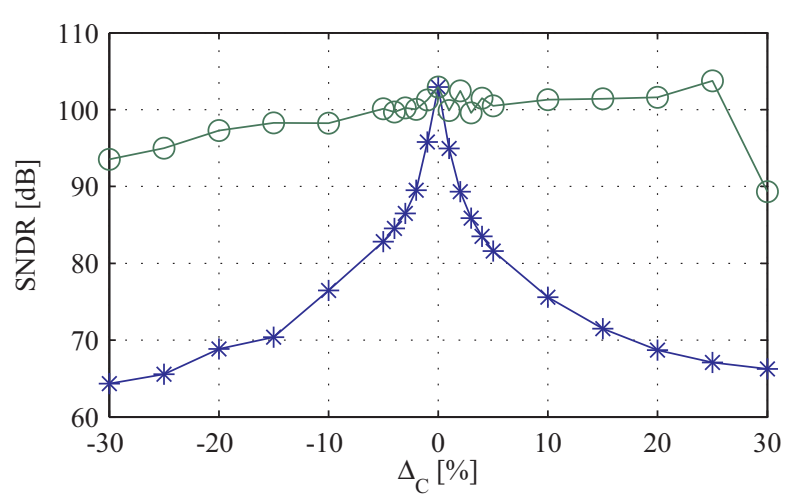

Figure 8: $\mathrm{ADC}_{\mathrm{ERI}} \mathrm{SNDR}$ performance vs. integrators coefficients deviation when using optimized $(\bigcirc)$ and unoptimized $(*)$ filter. $P_{\text {sig }}=-6 \mathrm{dBFS}$.

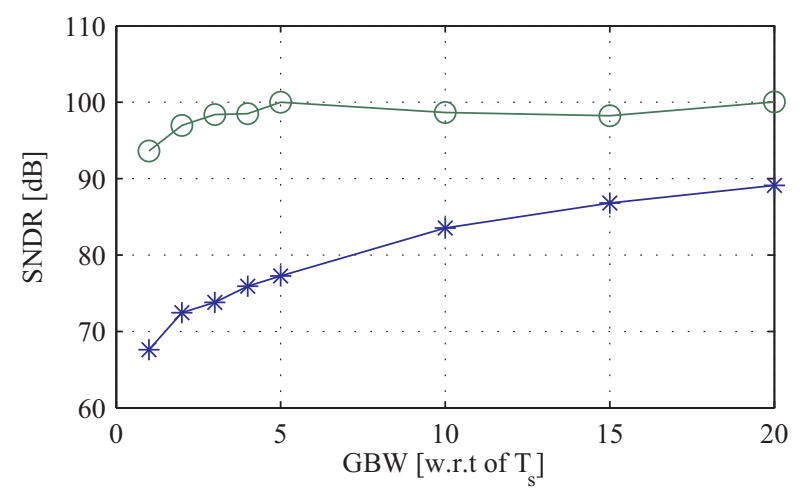

Figure 9: $A D C_{E R I}$ SNDR performance vs. integrators GBW product when using optimized $(\bigcirc)$ and unoptimized $(*)$ filter. $P_{\text {sig }}=-6 \mathrm{dBFS}$.

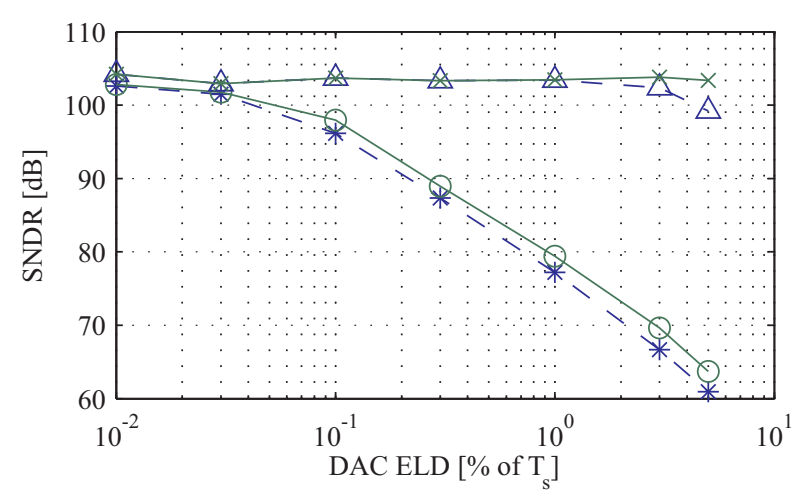

Figure 10: $\mathrm{ADC}_{\mathrm{ERI}}$ SNDR performance vs. DAC ELD when using optimized ('-') and unoptimized ('- - -') filter for different values of $\tau$. [ $* \& \bigcirc: \tau=1 / 10 T_{s}, \triangle \& \times: \tau=1 / 25 T_{s}$ ]. $P_{\text {sig }}=-6 \mathrm{dBFS}$.

samples coming from the $\Sigma \Delta$ modulator. This filter, when operates in transient mode, can instead be treated as an N-length finite impulse response (FIR) filter with the appropriate coefficients [17]. These coefficients can be obtained by computing 
the N-length impulse response of the transfer function in (1) and, in a system-level model with no non-idealities included, they allow a maximum quantization error refinement. Put it in another way, if one would assume an "infinite" resolution $\mathrm{ADC}_{\mathrm{ER}}$ in such model $\left(e_{Q E R}(n)=0\right)$, this filter would allow a complete cancellation of the I $\Sigma \Delta$ ADC quantization error, thus obtaining, an "infinite" resolution at the output of the ER-I $\Sigma \Delta$ ADC. Once non-idealities are inserted into the system, either by adding them at system-level or by advancing to a more refined level of abstraction, the transfer function in (1) becomes less effective, degrading the quantization error refinement and, as a consequence, the performance of the ER-I $\Sigma \Delta$ ADC. So as to maximize the I $\Sigma \Delta$ ADC's quantization error cancellation, this approach uses as objective the SNDR, assuming that a maximum SNDR will correspond to a maximum error refinement. Accordingly, the proposed filter uses the MATLAB optimization algorithm fmincon [18] to find the optimum $\mathrm{N}$ coefficients of the FIR filter so as to minimize the 1/SNDR value. Such algorithm attempts to find a constrained minimum of a scalar function, called the objective function, of several variables starting at an initial estimate. The coefficients obtained from the filter in (1) are then set as the initial estimate while the objective function calculates the SNDR of the filtered modulator's stream and returns the 1/SNDR value.

To validate the proposed filter, similar simulations as in Section 3 were performed to evaluate the $\mathrm{ADC}_{\mathrm{ERI}}$ output, with the exception of the sensitivity to clock jitter due to the random nature of this non-ideality. Moreover, the SNDR performance of the $\mathrm{ADC}_{\mathrm{ERI}}$ when the unoptimized filter derived from (1) is used has also been included for comparison.

As shown in Figure 8, the proposed filter can successfully counteract most of the degradation induced from the coefficients variations, obtaining up to $29 \mathrm{~dB}$ of SNDR improvement at $\Delta_{C}=-30 \%$. Although one can expect large deviations after physical implementation, the previous simulation highlights the possibility to compensate and even cancel the influence of such variations in the digital domain, as done in cascaded $\Sigma \Delta$ modulators. Alternatively, this method can also be used in the circuit and physical level implementation to optimize the digital filter in order to account for discrepancies between different abstraction levels .

The effect of the optimized filter when considering the finite amplifier GBW product is presented in Figure 9. Contrary to the respective simulation shown in Section 3, now it is enough to use an amplifier GBW product close to $f_{s}$ without suffering significant degradation. This represents a key feature for this architecture when compared to DT counterparts.

With respect to the ELD degradation, the proposed filter could only obtain marginal improvement, as depicted in Figure 10. The choice of the SCR-DAC mean lifetime value $\tau$ therefore represents a key design parameter to fully benefit from the extended range approach, not only due to its sensitivity to ELD but also due to clock jitter. Although not treated in this paper, it may also be possible to counteract jitter and ELD degradations by using multibit feed-back DAC. This approach, however, would increase the complexity of the digital filter and may require an extra calibration circuitry to reduce the DAC mis-

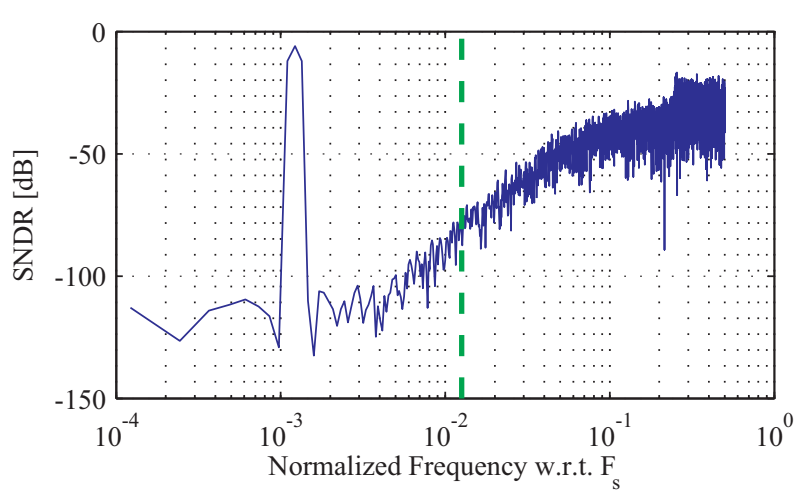

Figure 11: Power spectral density of the test case $\Sigma \Delta$ modulator output running continuously. $P_{\text {sig }}=-6 \mathrm{dBFS}$.

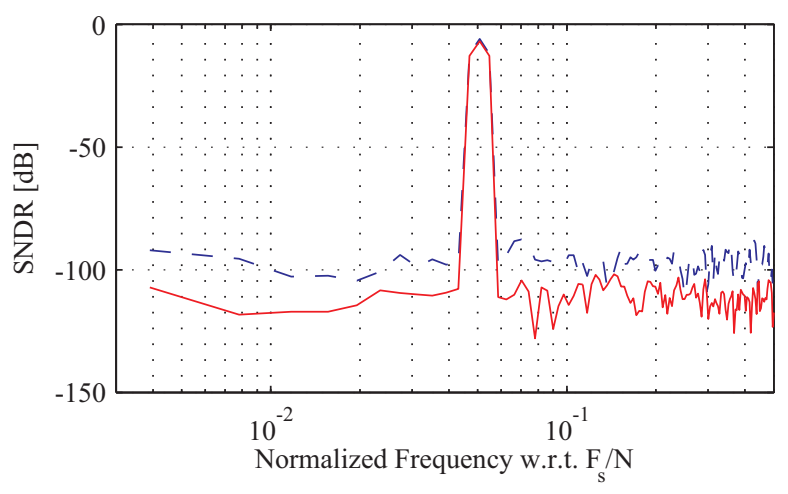

Figure 12: Power spectral density of the test case ER-I $\Sigma \Delta$ ADC with ('-') and without ('---') optimized filter. $P_{\text {sig }}=$ $-6 \mathrm{dBFS}$.

matches.

\section{Case study}

To validate the proposed architecture, a test case for an EEG digital recording system in accordance with the International Federation of Clinical Neurophysiology (IFCN) standard [19] is presented. The proposed case uses the architecture proposed in Section 2 and targets 13-bits resolution and $4 \mathrm{kSamples} / \mathrm{sec}$ so as to process 8 channels at $500 \mathrm{~Hz}$. Practical values for all previous non-idealities have been included and the digital filter described in Section 4 has been used to counteract their impact. The SCR-DAC uses a mean lifetime value $\tau=1 / 25 T_{\mathrm{s}}$ and a $5 \%$ of $T_{s}(312.5 \mathrm{~ns})$ delay was considered for the ELD. Moreover, a finite GBW product equal to $3 f_{s}$ and a coefficient deviation of $-10 \%$ was assumed for the integrators. Finally, a clock having a jitter standard deviation of $0.1 \%$ of $T_{s}(6.25 \mathrm{~ns})$ has also been included.

When the $\Sigma \Delta$ modulator is running continuously, it achieves an SNDR of $63.5 \mathrm{~dB} @-6 \mathrm{dBFS}$ as shown in Figure 11. Similarly, Figure 12 depicts the power spectral density (PSD) of the ER-I $\Sigma \Delta$ ADC with the optimized and unoptimized digital filter. The proposed ADC can achieve $83 \mathrm{~dB}$ SNDR (13.8 bits) when running for 40 cycles and using an 8-bit $\mathrm{ADC}_{\mathrm{ER}}$. A 13 
$\mathrm{dB}$ increment with respect to the unoptimized filter case is obtained. For comparison, the same architecture would require around 80 cycles in incremental mode to achieve similar performance. By using the extended range approach, it is therefore possible to reduce the number of cycles by $50 \%$ which would also reduce the GBW product of the integrators by $50 \%$ when compared to the CT I $\Sigma \Delta$ ADC. It is worth to notice that the 8bit $\mathrm{SAR} A \mathrm{ACC}_{\mathrm{ER}}$ would consume very little power with respect to the $\mathrm{ADC}_{\mathrm{I}}$ as it runs at the decimated frequency $f_{s} / N$.

\section{Conclusion}

The extended range approach in high-order SL CT I $\Sigma \Delta$ ADCs has been analyzed with the aid of a $3^{\text {rd }}$ order $\Sigma \Delta$ modulator as example. Behavioral simulations showed the high sensitivity of this topology to critical non-idealities when compared to CT I $\Sigma \Delta$ counterparts. As this sensitivity is mostly due to mismatches between the analog and digital transfer functions, a digital filter using optimization tools has been proposed to counteract their effect. This filter can effectively minimize the mismatches introduced by finite amplifier's GBW product as well as process variations. Simulation results show that the optimized digital filter can improve the ADC performance up to $13 \mathrm{~dB}$ when compared to the unoptimized filter case, further reducing the ADC's required number of cycles per conversion with respect to incremental counterparts.

\section{References}

[1] W. Yu, M. Aslan, and G. C. Temes, "82 dB SNDR 20channel incremental ADC with optimal decimation filter and digital correction," in IEEE Custom Integrated Circuits Conf., 2010, pp. 1-4.

[2] R. F. Yazicioglu, T. Torfs, P. Merken, J. Penders, V. Leonov, R. Puers, B. Gyselinckx, and C. V. Hoof, "Ultra-low-power biopotential interfaces and their applications in wearable and implantable systems," Microelectronics Journal, vol. 40, no. 9, pp. 1313 - 1321, 2009.

[3] M. Mollazadeh, K. Murari, G. Cauwenberghs, and N. Thakor, "From spikes to EEG: Integrated multichannel and selective acquisition of neuropotentials," in Annu. Int. Conf. of the IEEE Engineering in Medicine and Biology Society, 2008, pp. 2741-2744.

[4] J. Markus, J. Silva, and G. C. Temes, "Theory and applications of incremental $\Delta \Sigma$ converters," IEEE Trans. Circuits Syst. I, vol. 51, no. 4, pp. 678-690, 2004.

[5] G. Guo, D. Wu, Y. Shen, L. Pan, and J. Xu, “An optimal filter with optional resolution used in incremental ADC for sensor application," in IEEE Proc. Int. Conf. on Consumer Electronics, Communications and Networks, 2011, pp. 1042-1045.

[6] J. Uhlig, R. Schuffny, H. Neubauer, J. Hauer, and J. Haase, "A low-power continuous-time incremental 2nd-orderMASH $\Sigma \Delta$-modulator for a CMOS imager," in IEEE Int. Conf. on Electronics, Circuits and Systems, dec. 2009, pp. $33-36$.
[7] A. Agah, K. Vleugels, P. B. Griffin, M. Ronaghi, J. D. Plummer, and B. A. Wooley, "A high-resolution lowpower incremental $\Sigma \Delta$ ADC with extended range for biosensor arrays," IEEE J. Solid-State Circuits, vol. 45, no. 6, pp. 1099-1110, 2010.

[8] S. Pavan, N. Krishnapura, R. Pandarinathan, and P. Sankar, "A Power Optimized $\Sigma \Delta$ Continuous-Time ADC for Audio Applications," IEEE J. Solid-State Circuits, vol. 43, no. 2, pp. 351-360, 2008.

[9] G. C. Temes, "Micropower data converters: A tutorial," IEEE Trans. Circuits Syst. II, vol. 57, no. 6, pp. 405-410, 2010.

[10] M. Ortmanns, F. Gerfers, and Y. Manoli, "A continuoustime $\Sigma \Delta$ modulator with reduced sensitivity to clock jitter through SCR feedback," IEEE Trans. Circuits Syst. I, vol. 52, no. 5, pp. 875-884, 2005.

[11] J. Garcia and A. Rusu, "High-order continuous-time incremental $\Sigma \Delta$ ADC for multi-channel applications," in Proc. IEEE Int. Symp. Circuits and Systems, may 2011, pp. $1121-1124$.

[12] M. Ortmanns and F. Gerfers, Continuous-time sigma-delta A/D conversion: fundamentals, performance limits and robust implementations. Springer Verlag, 2006.

[13] L. Breems, R. Rutten, and G. Wetzker, "A cascaded continuous-time $\Sigma \Delta$ modulator with $67-\mathrm{dB}$ dynamic range in 10-MHz bandwidth," IEEE J. Solid-State Circuits, vol. 39, no. 12, pp. 2152-2160, 2004.

[14] M. Ortmanns, F. Gerfers, and Y. Manoli, "Compensation of finite gain-bandwidth induced errors in continuoustime sigma-delta modulators," IEEE Trans. Circuits Syst. I, vol. 51, no. 6, pp. 1088-1099, 2004.

[15] J. Steensgaard, Z. Zhang, W. Yu, A. Sarhegyi, L. Lucchese, D.-I. Kim, and G. C. Temes, "Noise-power optimization of incremental data converters," IEEE Trans. Circuits Syst. I, vol. 55, no. 5, pp. 1289-1296, 2008.

[16] S. Kavusi, H. Kakavand, and A. E. Gamal, "On Incremental Sigma-Delta Modulation With Optimal Filtering," IEEE Trans. Circuits Syst. I, vol. 53, no. 5, pp. 1004-1015, 2006.

[17] J. Markus, "High-Order Incremental Delta-Sigma Analog-to-Digital Converters," Ph.D. dissertation, Budapest University of Technology and Economics, 2005.

[18] Mathworks, "MATLAB R2011b fmincon documentation." [Online]. Available: http://www.mathworks.com/help/optim/ug/fmincon.html

[19] M. R. Nuwer et al., "IFCN standards for digital recording of clinical EEG," Electroencephalography and Clinical Neurophysiology, vol. 106, no. 3, pp. 259-261, Mar. 1998. 